\title{
Bilateral Ossiculoplasty in 1 Case of Achondroplasia
}

\author{
Jongyoon Jung ${ }^{1}$, Chulwon Yang ${ }^{2}$, Sunkyu Lee ${ }^{1}$ and June Choi ${ }^{1}$ \\ ${ }^{1}$ Department of Otorhinolaryngology-Head and Neck Surgery, Korea University Ansan Hospital, Ansan, \\ ${ }^{2}$ Department of Otorhinolaryngology-Head and Neck Surgery, Kyung Hee University Hospital at Gangdong, Seoul, Korea
}

\author{
Received July 8, 2013 \\ Revised September 5, 2013 \\ Accepted October 2, 2013
}

Achondroplasia is the most common skeletal dysplasia and it combines various complications with normal longevity. Hearing disturbance due to otitis media or an ossicular anomaly is one of the most common complications. Conductive hearing loss is suggested as the most common form of hearing loss. Temporal bone and middle ear structures are distorted in achondroplasia because of rotational change of the skull base. Authors experienced a case of an achondroplastic patient with bilateral hearing disturbance. We faced making a potential mistake during the previous operation but a favorable postoperative result occurred. Our experience could be helpful to other clinicians who face achondroplastic patients.

Korean J Audiol 2013;17:142-147

KEY WORDS: Achondroplasia · Conductive hearing loss · Temporal bone · Ear ossicles · Ossicular prosthesis.

\section{Introduction}

Achondroplasia is the most common of the short-limbed skeletal dysplasias, occurring in one in 26000 live births. ${ }^{1)}$ This autosomal dominant condition is caused by a mutation in fibroblast growth factor receptor 3 (FGFR3). ${ }^{2)}$ The basic defect in achondroplasia is a disturbance of endochondral ossification, and its consequences, such as short stature and midfacial hypoplasia, are typical features of achondroplasia. ${ }^{3)}$ Achondroplasia also has a variety of head and neck manifestations. The otolaryngologic complications include respiratory manifestations as a result of upper airway obstruction, ${ }^{4)}$ and frequent otitis media which may be accompanied by hearing loss and delayed speech. ${ }^{5)}$ Otitis media has been long recognized as a complication in achondroplasia, but little data have been published on the rates of otitis media and middle ear dysfunction in the achondroplasia population. High rates of otitis media have been considered secondary to the orientation and size of the Eustachian tube as well as impairment of nasal airflow and temporal bone abnormalities. ${ }^{5)}$ Although hearing loss in achondroplasia is mostly a conductive hearing loss, sensorineural

This is an Open Access article distributed under the terms of the Creative Commons Attribution Non-Commercial License (http://creativecommons org/licenses/by-nc/3.0/) which permits unrestricted non-commercial use, distribution, and reproduction in any medium, provided the original work is properly cited. hearing loss and mixed hearing loss are observable in achondroplasia. Conductive hearing loss can develop as a sequela of otitis media or ossicular anomalies because middle ear ossicles undergo endochondral ossification. ${ }^{3)}$ Sensorineural hearing loss can be expected in achondroplasia because of the sequelae of frequent/persistent otitis media or cochlear anomalies. ${ }^{6}$ The exact rate of hearing loss in achondroplasia has not been evaluated, and there is no dominant cause of hearing loss in achondroplasia.

The authors experienced 1 case of an achondroplastic patient who complained of bilateral hearing loss, and we evaluated the patient with serial audiologic examinations. In the serial examinations, the pattern of hearing disturbance changed from mixed hearing loss to conductive hearing loss. Also, the authors corrected the conductive hearing loss with an operation and got a fair surgical result. So we report our experience, with a review of the references.

\section{Case Report}

A 12 year-old female patient suffering from persistent hearing loss after birth visited the outpatient clinic for evaluation and treatment of the hearing loss. The patient showed typical features of achondroplasia, such as short limbs, especially the proximal segment, with a long trunk, narrow thorax, large 
head with frontal bossing, and midfacial hypoplasia at birth. Although there was no one with achondroplasia in the family, and a genetic study of FGFR3 was not performed, the typical features of achondroplasia in the patient and the short statue were enough to diagnose the achondroplasia. The patient had suffered from frequent otitis media since childhood, and had a history of bilateral tympanostomy tube insertion 7 years ago. Both tympanostomy tubes were extruded 2 years after insertion. Because she showed a feature of sensorineural hearing loss, she was referred to our hospital for consultation for hearing rehabilitation, such as the use of a hearing aid.

The right tympanic membrane was intact and there was a large perforation in the left tympanic membrane. Pure tone audiometry (PTA) was performed and the hearing threshold averaged from $0.5 \mathrm{kHz}, 1 \mathrm{kHz}, 2 \mathrm{kHz}$, and $4 \mathrm{kHz}$. The air conduction hearing threshold was $82 \mathrm{~dB}$ HL on the right and 52 $\mathrm{dB} H \mathrm{HL}$ on the right, and the bone conduction hearing threshold was $46 \mathrm{~dB}$ HL on the left, and $44 \mathrm{~dB}$ HL on the left. The type of hearing loss was mixed hearing loss (Fig. 1A). Speech recognition threshold and the speech discrimination score were $70 \mathrm{~dB} \mathrm{HL}$ and $96 \%$ on the right, and $50 \mathrm{~dB} \mathrm{HL}$ and $100 \%$ on the left, respectively. On auditory brainstem response testing, there was prolonged interlatency of the right side, while the left side was normal (Fig. 2). The patient was recommended to use a hearing aid on the right side and have tympanoplasty for the left side. But the patient denied further treatment and was lost.

The patient re-visited the outpatient clinic 5 years later after the first visit. The right ear had been operated at another hospital but she still complained of hearing loss on the right side, and she suffered from intermittent otorrhea and otalgia of the left ear. There were no specific findings of the right ear. The tympanic membrane of the left ear was perforated and the margin of the perforation was attached to the mucosa of the middle ear. Air conduction and bone conduction hearing threshold were $65 \mathrm{~dB}$ HL and $17 \mathrm{~dB}$ HL for the right ear, and $30 \mathrm{~dB}$ $\mathrm{HL}$ and $12 \mathrm{~dB}$ HL for the left ear, respectively (Fig. 1B). Temporal bone computed tomography was performed. The right ear was well pneumatized, and was in a canal-wall-up mastoidectomized state. There was no incus and malleus head at the epitympanum, and foreign material, estimated to be material of the implanted prosthesis, was visible in the mesotympanum. There was no abnormal lesion in the middle ear cavity. Although the left ear was well pneumatized, a lesion with soft tissue attenuation partially filled the mastoid air cells. The incus and malleus were normal in appearance (Fig. 3).
Fig. 1. Results of preoperative pure tone audiometry (PTA). A: Initial PTA showed a type of bilateral mixed hearing loss. B: Second PTA showed a type of bilateral conductive hearing loss.

Fig. 2. Results of auditory brain stem response. Latency of the right side was prolonged, but interlatency was normal. Interaural latency of the $\mathrm{V}$ wave was normal.
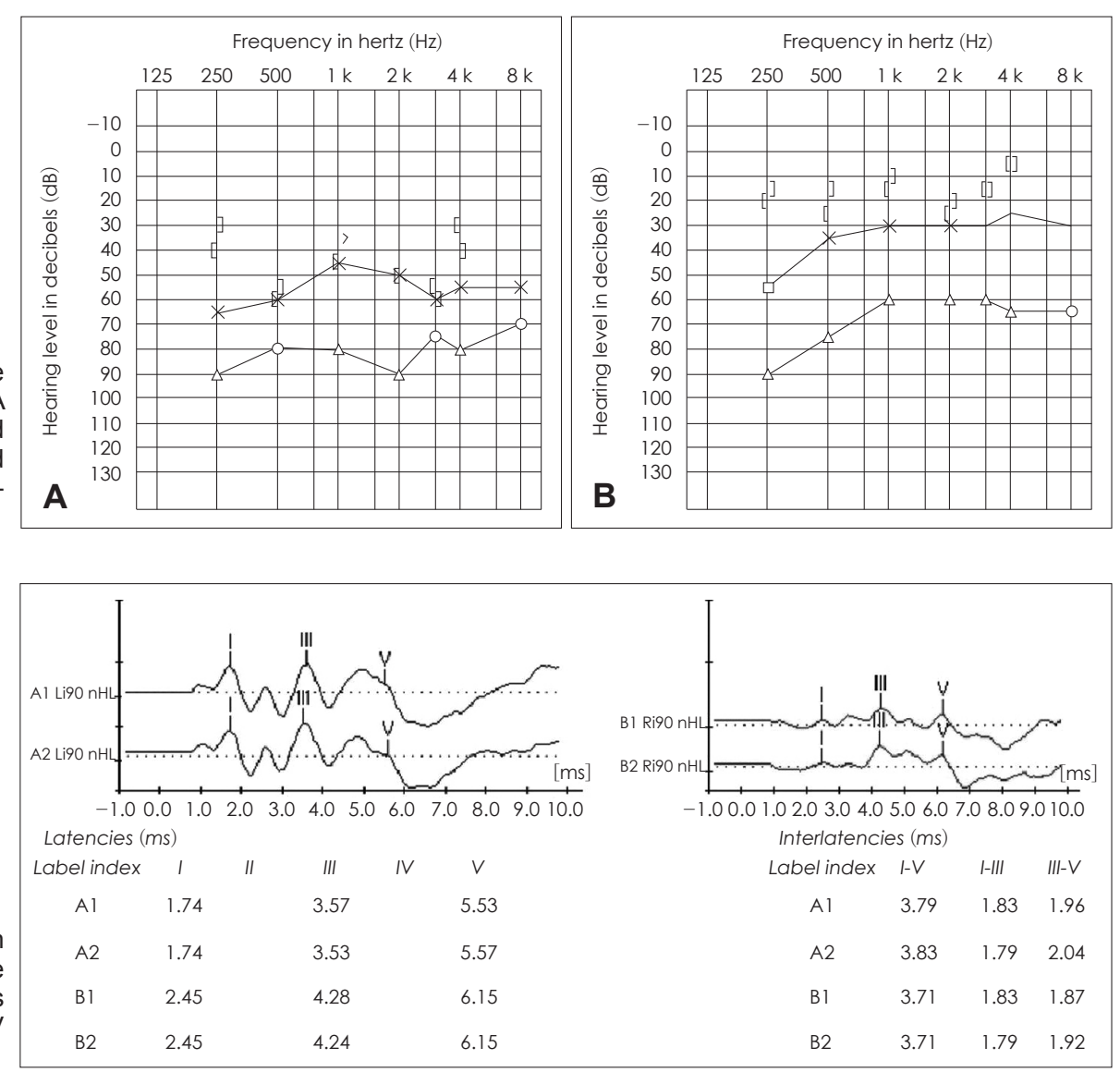
Right exploratory tympanotomy was planned first because the air-bone gap was larger and the state of the tympanic membrane was better than in the left ear. A previously inserted total ossicular replacement prosthesis (TORP) was found dur- ing the operation, and the TORP was displaced from the stapes footplate. Curetting of the posterior-superior portion of the bony tympanic ring was done and a stapedial superstructure was observed. All stapedial superstructures were intact and locat-
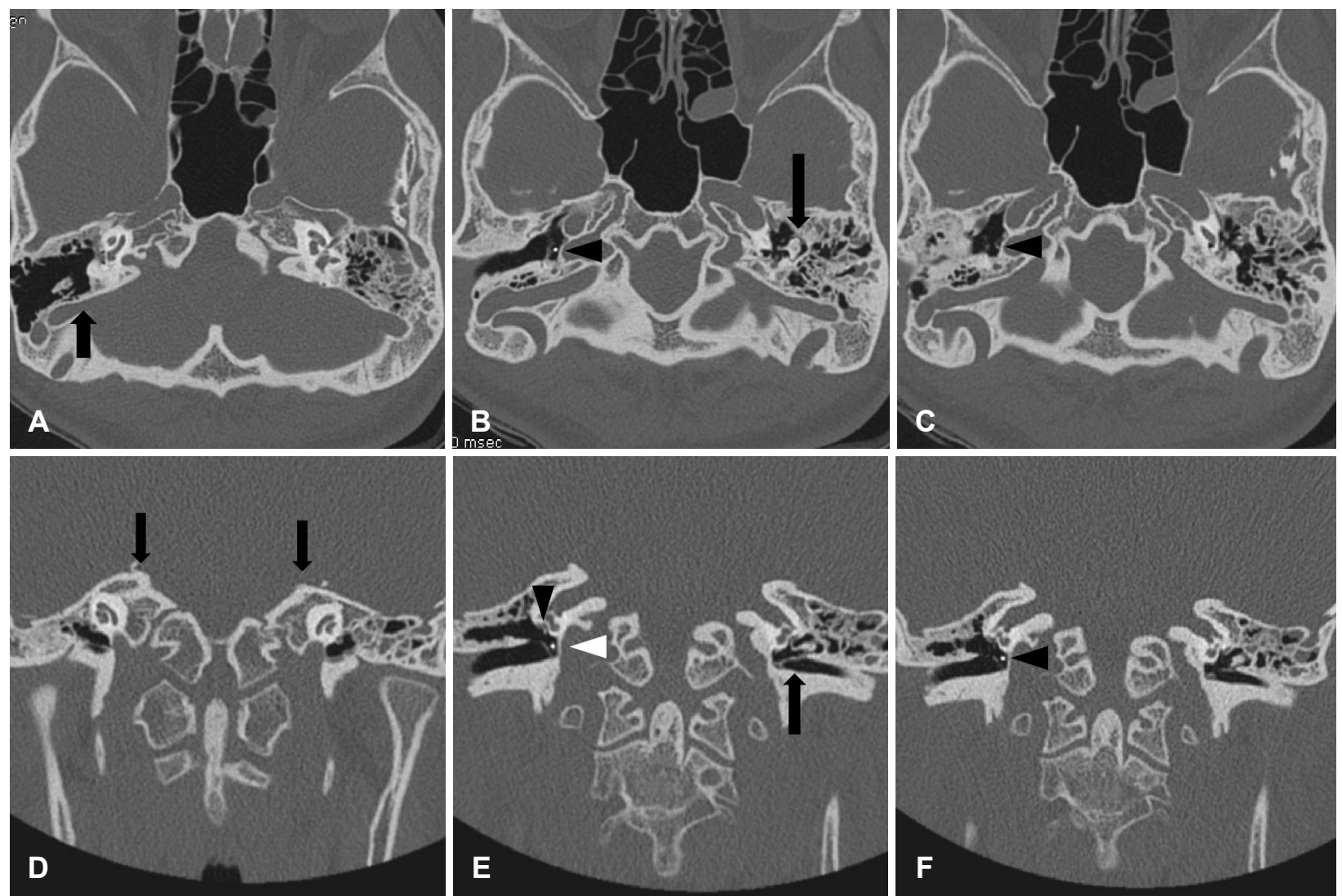

Fig. 3. Temporal bone computed tomography. A: Axial image. Previously performed canal-wall-up mastoidectomy state (black arrow). Left side mastoid cavity is relatively well-pneumatized with minimal soft tissue density lesion. B: Axial image. Previously implanted prosthesis is visible at the right tympanic cavity (black arrow head). Malleo-incudal complex appears together as broad ice-cream cone because of rotation into axial plane (black arrow). C: Axial image. Stapes superstructure (black arrow head) is observed and no prosthesis is attached to stapes superstructure. D: Coronal image shows towering petrous bones (black arrow). E: Coronal image. Stapes superstructure (black arrow head) and previous implanted prosthesis (white arrow head) is visible. Displacement of prosthesis can be observed. Oval window faces almost directly inferiorly. Scutum is directed toward inferior aspect of promontory (black arrow). F: Coronal image. Displaced implanted prosthesis (black arrow head).
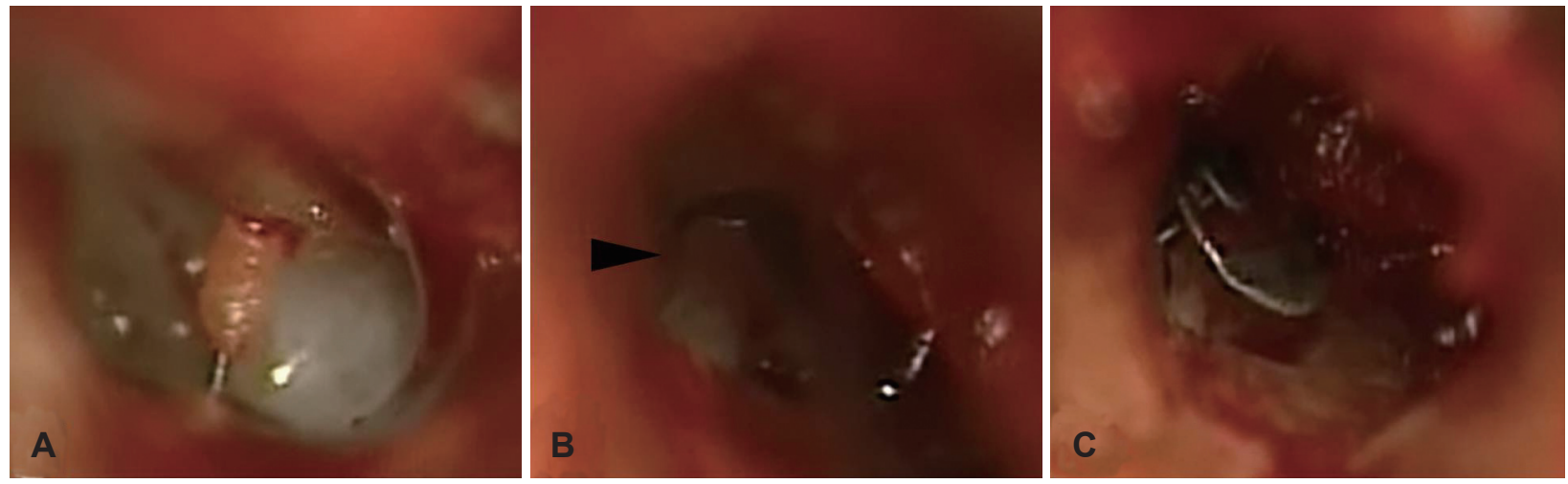

Fig. 4. Intraoperative findings of the right ear. A: The previously implanted total ossicular replacement prosthesis was visible and its location was displaced from the stapes footplate. B: Head of the stapes (black arrowhead) was found and palpated with an instrument. C: Partial ossicular replacement prosthesis was implanted on the stapes head. 

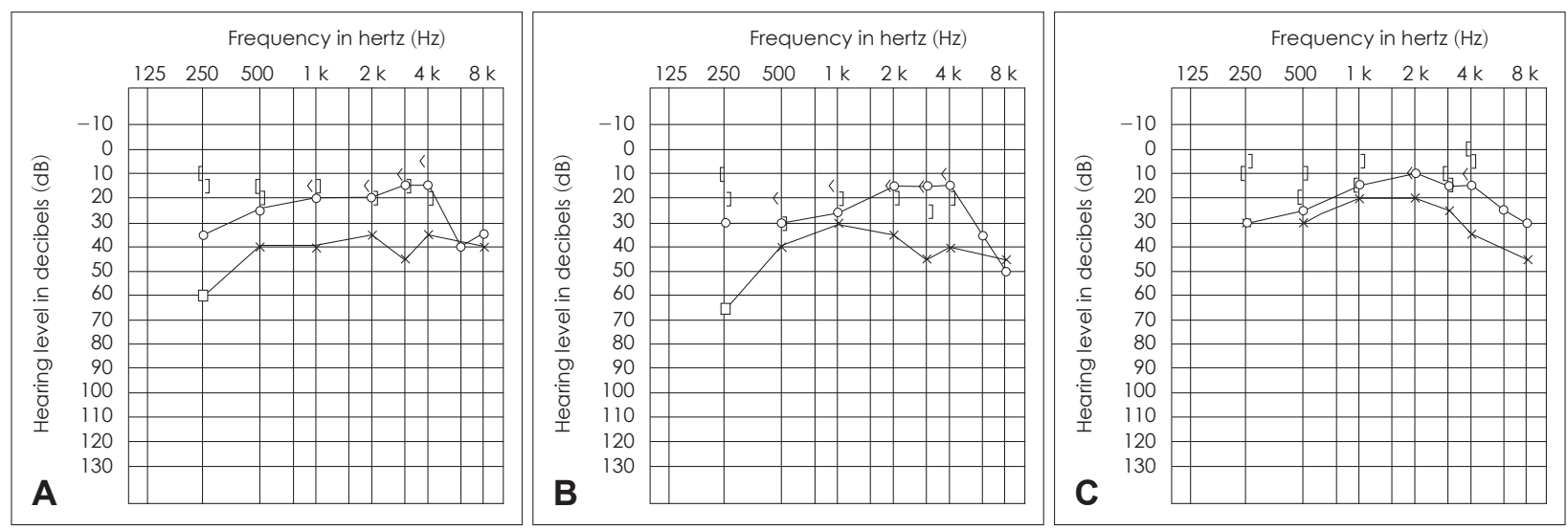

Fig. 5. Results of postoperative pure tone analysis (PTA). A: PTA 1 month after the right ear operation. Air-bone gap of the right side was decreased. B: PTA 3 months after the right ear operation. The corrected air-bone gap remained. C: PTA 6 months after the right ear operation and 3 months after the left ear operation. Hearing on the right side was maintained well and the air-bone gap of the left ear was decreased.

ed at the posterior-superior side of the middle ear cavity. A round window reflex during stapes palpation was observed. A partial ossicular replacement prosthesis (PORP) made of titanium was inserted on the stapes head and the conchal cartilage was interpositioned between the tympanic membrane and prosthesis head (Fig. 4). Gelfoam was packed into the external auditory canal, but not in the middle ear cavity. The whole of the gelfoam was removed two weeks after the operation, and postoperative PTA was performed four weeks after the operation: the hearing threshold was $22 \mathrm{~dB}$ HL on the right, and $38 \mathrm{~dB}$ HL on the left (Fig. 5A). A second PTA was performed three months after the operation, and the hearing threshold was $21 \mathrm{~dB}$ HL on the right, and $36 \mathrm{~dB}$ HL on the left (Fig. 5B).

After confirmation of correction of hearing in the right ear, an operation on the left ear was planned. During the operation on the left ear, a large sized central perforation with adhesion of the perforation margin to the middle ear mucosa was observed. Although all ossicles were normal and their continuity was intact, the mobility of the ossicles was decreased during palpation. The incudostapedial joint was separated and the distal portion of the long process of the incus was removed because of decreased motility. The PORP was interpositioned between the stapes head and the handle of the malleus, and the conchal cartilage was also interpositioned on the prosthesis head to avoid contact of the prosthesis with the tympanic membrane. The temporalis muscle fascia was inserted via the underlay method, and gelfoam was packed into the middle ear cavity and external auditory canal. The gelfoam was removed on the postoperative 14th day. PTA was performed three months after the second operation, and the hearing threshold was $16 \mathrm{~dB}$ HL on the right, and $26 \mathrm{~dB}$ HL on the left (Fig. 5C). The patient was observed at the outpatient clinic.

\section{Discussion}

Achondroplasia is the most common skeletal dysplasia and is an autosomal-dominant disorder with essentially complete penetrance. ${ }^{2)}$ The achondroplasia locus is mapped to chromosome 4 p16.3 and a recurrent heterozygous mutation of FGFR3 has been identified. ${ }^{7)}$ Although penetrance of the mutation is complete, more than $85 \%$ of patients are born from unaffected parents and this exceptional occurrence is found to be due to mosaicism in the father's sperm. ${ }^{8)}$ Increased paternal age has been described as a factor. ${ }^{8}$

The basic defect in achondroplasia is disturbance of endochondral ossification, and the result is selective deficiency of bone growth in long bones. ${ }^{3)}$ In the temporal bone, only the petrous part arises entirely in the cartilage. The squamous part, most of the mastoid portions, tympanic cavity proper and eustachian tube develop in membranous ossification. Among the three ossicles, the malleus and incus are accepted to derive from the first branchial arch, maturing by way of the continuing deposition of endosteal bone during the course of fetal life, similar to long bone growth in the appendicular skeleton. The stapes has a more complex development, having a dual origin from the branchial cartilage and cartilage from the otic capsule (which contributes to the footplate). ${ }^{3)}$ These developmental changes produce the typical findings of the temporal bone in achondroplasia: 1) towering of the petrous ridge and a high jugular bulb; 2) narrowing of the skull base; and 3) relative rotation of the cochlea and other temporal bone structures (Fig. 3).

Otitis media has been recognized as a complication in achondroplasia, ${ }^{9)}$ but there are few published data on the incidence of this problem. Hall apparently found that $75 \%$ of 150 patients in an unpublished series of patients with achondroplasia had suffered otitis media and that the rate fell to $11 \%$ over the age 
of 18 years. ${ }^{10)}$ Berkowitz, et al. ${ }^{5)}$ used the indirect measure of the insertion of ventilation tubes to assess the impact of otitis media and found that 33 of 61 patients $(54 \%)$ had had tubes inserted. Hunter, et al. also reported the high rate of otitis media in achondroplastic patients (95\% under age of 5 years) and the high rate of insertion of ventilation tubes ( $78 \%$ under age of 10 years). The high rate of otitis media has been considered secondary to the short and wide Eustachian tube, impaired nasal airflow, and temporal bone abnormalities. ${ }^{5,11)}$

Prospective studies have not been reported until now, and longitudinal, cumulative data do not exist, either. The frequency of tympanostomy tube insertion was used to analyze the incidence of otitis media in the previous studies, and so it could not reflect the exact incidence of otitis media. Also otitis media occurred frequently in the normal control children. A more precise prospective study would be needed in the study on the rate of otitis media in achondroplastic patients.

Hearing loss is common in individuals affected by skeletal dysplasia, such as achondroplasia. Glass, et al. ${ }^{12)}$ reported hearing loss of $>20 \mathrm{~dB}$ in $60.7 \%$ of 29 patients with achondroplasia. Stura, et al. ${ }^{6)}$ found hearing loss in $55.5 \%$ of 18 patients with achondroplasia. Collins and $\mathrm{Choi}^{13)}$ found conductive hearing loss in $68 \%$ of the audiologic records of children with achondroplasia.

Hearing loss in achondroplasia can be conductive, sensorineural, or mixed hearing loss. Conductive hearing loss in achondroplasia is the most dominant form of hearing loss and the causes are most likely to be middle ear dysfunction or ossicular chain stiffness, either congenital or acquired owing to past chronic middle ear disease. Previously, abnormal structure of the temporal bone was suggested as the cause of Eustachian tube dysfunction. But a prior study showed no evidence of poor middle ear drainage, using high resolution temporal bone computed tomography (TBCT). ${ }^{3)}$ It was also revealed that there was no correlation between $\mathrm{CT}$ findings of the temporal bone and the type or severity of hearing loss. ${ }^{14)}$ Although an ossicular anomaly could be a possible cause regarding the development of the ossicles (especially the malleus and incus), almost all studies reported the normal appearance of ossicles in achondroplastic patients. Sensorineural hearing loss could be caused by cochlear malformations; however, high-resolution CT would be not sufficient to evaluate cochlear function, and there will be a need for further studies.

In this report, the longitudinal hearing threshold could be evaluated. Bilateral mixed hearing loss was observed at the first visit and the type of hearing loss changed to bilateral conductive hearing loss at the second visit (Fig. 1). Recurrent otitis media and its effects could be the cause of permanent sensorineural hearing loss in achondroplasia. In this case, the sensorineural component was improved at the second visit, so the effects of the otitis media may not have been a cause of the sensorineural hearing loss. Swelling of the middle ear mucosa or the presence of granulation tissue around the oval window/round window could be the cause of the sensorineural hearing loss in otitis media, and the threshold of bone conduction can be improved when these factors are corrected by operation. Also slow development of the temporal bone can be the cause of the inappropriate examination of the bone conduction threshold. Although the exact cause of changes of the bone conduction threshold could not be identified, it may have been due to improving conditions of the middle ear or the late development of the mastoid.

Conductive hearing loss was observed at the second visit and the cause of the conductive hearing loss was displacement of the prosthesis. The initial ossicular state could not be evaluated to determine whether a congenital ossicular anomaly or secondary ossicular stiffness existed. In achondroplasia, structural abnormalities of the temporal bone can develop because of rotation of the medial structure. The cochlea was upward rotated and the scutum was directed well below the promontory and basal turn of the cochlea. Only the most inferior aspect of the promontory can be observed through the external auditory canal. Also, rotational changes affect the vestibule and related structures, so the oval window faces almost directly inferiorly. It can make it difficult to find the stapes and its superstructure during an operation. Adequate analysis of the TBCT before an operation, and procedures for visualization of the middle ear, such as canaloplasty, would be needed during an operation. In this case, curetting of the posterior-superior bony tympanic ring was used for visualization of the stapes head. Also drilling of the same portion of the external auditory canal could be useful for visualization of the stapes and care should be taken not to damage the tympanomeatal flap during drilling. A small piece of aluminium foil (packing material of vicryl string) can be used as a protector during the drilling.

Perforation of the tympanic membrane, adhesion of the perforation margin to the middle ear mucosa and stiffness of the ossicles were observed in the left ear. These changes may have been due to the previous tympanostomy tube insertion and the frequent middle ear infections. Despite lack of ossicular mobility, the air-bone gap was $18 \mathrm{~dB} \mathrm{HL}$, and only tympanoplasty would have been effective. The purpose of surgery is correction of the conductive hearing loss, and it was decided to correct the whole possible cause of the hearing loss during the operation. Malleo-stapedial interposition can preserve physiologic sound transmission and has shown a $66-85 \%$ success rate in many reports. Adequate fitting of the prosthesis 
between the malleus handle and stapes head was possible during the operation, and ossiculoplasty rather than only tympanoplasty was performed.

Although a congenital ossicular anomaly could be possible considering the development of the ossicles, the incidence of ossicular anomaly is low. Acquired ossicular stiffness can develop when repeated, persistent middle ear disease accompanies it and it can be the cause of conductive hearing loss. Normal ossicular continuity and acquired ossicular stiffness should be considered before suspecting an ossicular anomaly based on abnormal looking ossicles in the TBCT, and sufficient evaluation and verification are needed before surgical treatment.

Although this is just one case report, it shows a typical finding of achondroplasia and a way of correcting the hearing loss in achondroplasia. It could be helpful to many clinicians who meet achondroplastic patients for choosing the appropriate surgical treatment.

\section{REFERENCES}

1) Oberklaid F, Danks DM, Jensen F, Stace L, Rosshandler S. Achondroplasia and hypochondroplasia. Comments on frequency, mutation rate, and radiological features in skull and spine. J Med Genet 1979;16:140-6.

2) He L, Shobnam N, Wimley WC, Hristova K. FGFR3 heterodimerization in achondroplasia, the most common form of human dwarfism. J Biol Chem 2011;286:13272-81.

3) Cobb SR, Shohat M, Mehringer CM, Lachman R. CT of the tempo- ral bone in achondroplasia. AJNR Am J Neuroradiol 1988;9:1195-9.

4) Reid CS, Pyeritz RE, Kopits SE, Maria BL, Wang H, McPherson $\mathrm{RW}$, et al. Cervicomedullary compression in young patients with achondroplasia: value of comprehensive neurologic and respiratory evaluation. J Pediatr 1987;110:522-30.

5) Berkowitz RG, Grundfast KM, Scott C, Saal H, Stern H, Rosenbaum K. Middle ear disease in childhood achondroplasia. Ear Nose Throat J 1991;70:305-8.

6) Stura M, Boero S, Origo C, Tarantino V. Evaluation of hearing in achondroplastic patients. Basic Life Sci 1988;48:183-6.

7) Rousseau F, Bonaventure J, Legeai-Mallet L, Schmidt H, Weissenbach J, Maroteaux P, et al. Clinical and genetic heterogeneity of hypochondroplasia. J Med Genet 1996;33:749-52.

8) Murdoch JL, Walker BA, Hall JG, Abbey H, Smith KK, McKusick VA. Achondroplasia--a genetic and statistical survey. Ann Hum Genet 1970;33:227-44.

9) Cohen MM Jr. Dysmorphic growth and development and the study of craniofacial syndromes. J Craniofac Genet Dev Biol Suppl 1985;1: 43-55.

10) Hall JG. Human achondroplasia. A multidisciplinary approach. In: Nicoletti B, Kopits SE, Ascani E, Mckusick VA, editors. The natural history of achondroplasia. New York: Plenum Press;1988. p.3-9.

11) Cohen MM Jr, Walker GF, Phillips C. A morphometric analysis of the craniofacial configuration in achondroplasia. J Craniofac Genet Dev Biol Suppl 1985;1:139-65.

12) Glass L, Shapiro I, Hodge SE, Bergstrom L, Rimoin DL. Audiological findings of patients with achondroplasia. Int J Pediatr Otorhinolaryngol 1981;3:129-35.

13) Collins WO, Choi SS. Otolaryngologic manifestations of achondroplasia. Arch Otolaryngol Head Neck Surg 2007;133:237-44.

14) Shohat M, Flaum E, Cobb SR, Lachman R, Rubin C, Ash C, et al. Hearing loss and temporal bone structure in achondroplasia. Am J Med Genet 1993;45:548-51. 